\title{
Il cinema «idea» di Eduardo
}

\author{
Gino Frezza
}

\begin{abstract}
Eduardo nel cinema è stato attore, sceneggiatore, regista. Il saggio di Frezza ricostruisce la sua poliedrica attività cinematografica dagli anni trenta agli anni sessanta, anche in relazione/opposizione con l'opera e la statura filmica del fratello Peppino; la particolare maniera di Eduardo di essere attore nel cinema diviene, in una riflessione dedicata proprio al modo d'essere attori sullo schermo da parte di Orson Welles, occasione privilegiata, estremamente illuminante, per chiarire le caratteristiche problematiche di un ruolo chiave della comunicazione filmica.
\end{abstract}

Parole chiave: attore, schermo, Eduardo.

\begin{abstract}
In the cinema Eduardo was an actor, scriptwriter and director. The Frezza paper reconstructs his multifaceted cinematographic activities from the 30 s to the 60 s also in relation/opposition to the work and film stature of his brother Peppino. It also explores the particular way in which Eduardo is an actor in cinema becomes, in reflecting on what is an actor on the Orson Wells scale, a privileged occasion, extremely enlightening in terms of the problematic characteristics of a key role of film communication.
\end{abstract}

Key words: actor, screen, Eduardo.

Eduardo De Filippo è una presenza complessa nell'ambito del cinema e, specificamente, di quello italiano. Attore di primissimo rilievo già dagli anni trenta, inizia la sua carriera in forte solidarietà con il fratello Peppino e con la sorella Titina (la quale nel cinema ha una presenza più ridotta di quella dei due fratelli).

Eduardo e Peppino già dal loro esordio, e poi per tutta la prima parte — gli anni trenta - della loro carriera nel cinema, sono difficilmente scindibili l'uno dall'altro, anche se già dal secondo film — che li impone attori di notevole successo nelle sale- se ne distingue la diversa fisionomia e la differente colloca- 
zione entro le strategie comunicative di un medium che, per i due fratelli, non è così importante come, invece, il teatro.

Nelle loro dichiarazioni, sia Eduardo che Peppino considerano il cinema un luogo espressivo quasi di ripiego, di «sopravvivenza», di secondo e minor grado d'importanza rispetto al palcoscenico, che considerano la vera arena di attività, di investimento creativo, di sperimentazione recitativa, sulla quale ritengono valga la pena di affermare una visione artistica. Il cinema - per i due fratelli- è uno spazio che consolida l'immagine verso un pubblico che gradisce le loro performance; l'attività cinematografica rende maggiormente permanente e stabile una fisionomia artistica che, principalmente, afferma una linea di continuità e di innovazione nel repertorio teatrale del Novecento. Di fronte a questa opinione che i fratelli De Filippo esprimono sul cinema, nei confronti della loro duplice carriera (fra scena e schermo) occorre distinguere, di conseguenza, due piani dell'analisi: una cosa è ciò che essi dicono di se stessi nel cinema; un'altra è ciò che in realtà sono e fanno nel cinema.

Ciò che i De Filippo dicono vale a stabilire quel che essi pensano del cinema, o meglio ciò che vi proiettano in termini di progetto, ambizione e traguardo; si tratta di una visione complessa, a tratti difficile, a tratti di chiara sottovalutazione, e qualche volta di parco apprezzamento. Ma le idee che gli artisti esprimono sulle proprie sfere di attività sono parziali, raccolgono una angolazione talvolta sofferta e quindi limitata alla specifica esperienza vissuta dall'interno. In altre parole, questa visione personale può servire a chi indaga dall'esterno, ma talvolta incappa in errori, in clamorosi abbagli.

Importa piuttosto ciò che l'artista compie, l'opera che, prendendo forma, si dispone a essere giudicata e osservata e consumata o goduta dai fruitori. In tal senso è la concreta attività filmica di Eduardo e Peppino che fornisce chiavi atte a comprendere l'opera di entrambi, e in particolare consente di stringere un angolo di osservazione perspicace su Eduardo. Per varie ragioni, che riassumo nei seguenti punti:

1. Eduardo vive la prima parte della sua carriera cinematografica soprattutto da attore, assieme al fratello Peppino e alla sorella Titina; in questa fase non secondaria della loro attività, è già possibile riconoscere la diversa posizione che i due fratelli assumono nei confronti del medium filmico e delle sue caratteristiche; ma una attenta ricognizione di questa prima parte della carriera filmica di Eduardo (in questa fase, non scindibile da quella di Peppino) deve operare una nuova, accorta, valutazione rispetto a quelle fatte da attestate esplorazioni storico-cinematografiche. Di solito infatti i due fratelli sono ritenuti in grado di apportare nel cinema degli anni trenta le linee di un teatro moderno contemperato da un lato dalla tradizione ottocentesca partenopea (Petito, Scarpetta, ecc.), dall'altro da un autore come Roberto Bracco e dall'altro, ancora, da Pirandello (al quale i fratelli sono legati da forte amicizia e stima).

$\mathrm{Ma}$, a queste valutazioni, occorre aggiungere altri aspetti. Peppino e Eduardo intervengono nel cinema italiano in un decennio cruciale, che si 
presenta diviso, scisso in correnti opposte; da un lato le linee autarchiche di controllo e di censura del regime fascista, che puntano a film irregimentati in celebrazioni o esiti propagandistici, e dall'altro le spinte di rinnovamento culturale e professionale, estetico-espressivo, riconducibili a vari personaggi: da Emilio Cecchi a Alessandro Blasetti, Mario Camerini, Ferdinando Poggioli e a quegli autori (Matarazzo, Mattoli, Bragaglia, Palermi, ecc.) che riprendono le fila di un cinema dei generi di alto profilo popolare e comunicativo. In questa situazione, i De Filippo risultano fattori integrati nel decisivo rinnovamento del nostro cinema, che si accentua nella seconda metà del decennio e configura una sfida d'intelligenza e di maturità espressiva che cerca di fuoriuscire (spesso riuscendo) dalle strategie di controllo e di uniformità ideologica al regime fascista. Per gran parte, tale cinema si differenzia dalla cultura autarchica del fascismo grazie a uno spirito di libertà narrativa e di coraggio tematico che si manifesta in termini non solo metaforici.

Come attore, Eduardo assieme a Peppino fornisce un contributo rilevante a tale fase creativa del cinema italiano, con precise e positive conseguenze su quella del primo dopoguerra. $\grave{E}$ un contributo che accomuna i due fratelli a diverse figure della commedia popolare di quel decennio: non solo attori come Gino Cervi, Vittorio De Sica, Armando Falconi, Sergio Tofano, e naturalmente Totò, ma registi e autori, da Mario Camerini a Raffaello Matarazzo, Mario Bonnard, Giuseppe Amato (in veste di produttore e anche di regista), Esodo Pratelli, Carlo Ludovico Bragaglia, Mario Mattòli, Gennaro Righelli (e vi aggiungerei la solidarietà produttiva con una figura del livello di Raffaele Colamonici). Di questa necessaria rivalutazione l'ambiente degli studiosi e degli storici del cinema è consapevole da oltre un decennio, e basti qui ricordare alcuni essenziali studi, da quelli di Francesco Savio a quelli di Gian Piero Brunetta, Mino Argentieri, Adriano Aprà e Patrizia Pistagnesi. ${ }^{1}$

2. Eduardo già dagli anni trenta ha verso il cinema un rapporto di professionale curiosità. Si potrebbe dire di "estrema consapevolezza», se non fosse che l'apprendere, da parte sua, le logiche e i congegni comunicativi del cinema avviene attraverso un rilevante distacco. In realtà egli non si proietta mai fino in fondo nel cinema né vi identifica uno spazio creativo nel quale spingere, tutt'intera, la propria sensibilità artistica. ${ }^{2}$ Niente di male, intendiamoci, né questa valutazione va intesa come un giudizio negativo.

1. Sul cinema italiano di quel periodo, rivisitato in una accorta prospettiva storico-critica, cfr. F. SAVIO, Ma l'amore no, Milano: Sonzogno, 1975; ID., Cinecittà anni trenta, Roma: Bulzoni, 1979, 3 vol.; G. P. BRUNETTA, Storia del cinema italiano, vol. I: $1895-1945$ e vol. II: Dal 1945 agli anni ottanta, Roma: Editori Riuniti, 1979-1982; M. ARGENTIERI (a cura di), Risate di regime. La commedia italiana 1930-1944, Venezia: Marsilio, 1991; A. APRÀ (a cura di), Materiali sul cinema italiano 1929/43, Pesaro: Mostra Internazionale del Nuovo Cinema, 1975; A. APRÀ, P. PISTAGNESI (a cura di), Nuovi materiali sul cinema italiano 1929/43, Pesaro, Mostra Internazionale del Nuovo Cinema, 1976, 2 vol.

2. In F. SAVIO, Cinecittà..., cit., p. 409, ad una precisa domanda che tende a chiarire il rapporto che Eduardo ha verso il cinema rispetto al teatro, questi risponde: "L'ho fatto seriamente: ha visto Napoli Milionaria? Però, però, caro amico, fortuna che esiste il teatro.» 
Eduardo si avvicina al cinema considerandolo un mezzo di comunicazione dal quale, certamente, ritiene di imparare qualcosa di utile, con il quale intende fare i conti e provare la sua adattabilità espressiva; il cinema per lui è un medium con il quale tentare l'opportunità di un contatto con il grande pubblico delle sale, ma sempre con uno sguardo teso a trasferire i contenuti creativi da Eduardo stesso elaborati in altro ambito, cioè a trovare delle equivalenze possibili con il teatro, che giustifichino la scelta di dedicarvi tempo e attenzione.

In una importante intervista con Francesco Savio, ${ }^{3}$ Eduardo liquida buona parte del cinema interpretato col fratello Peppino nell'anteguerra e nei primi anni del conflitto come "teatro fotografato"; la locuzione adoperata non è sostanzialmente sbagliata, ma va spogliata di ogni limite negativo. Quei film sono «teatro fotografato» ma con segni e marcature che trapiantano nel cinema una leggerezza decisamente apprezzabile, e che livella queste prove giovanili a una sfera di maturità creativa. Inoltre questa parte dell'opera di Eduardo e di Peppino svela alcune sotterranee continuità con le successive opere filmiche, teatrali e televisive. Il loro è un cinema teatrale non pedante e non forzato, non appesantito, depurato di ogni pomposità, ravvicinato alle meccaniche e alla dinamicità della visione sonora filmica. È preferibile, forse, la dizione di «teatro filmico essenziale», che definisce una forma di cinema, professionalmente trasferita dal teatro alla pellicola, che si avvale di una puntuale cognizione delle differenze fra palcoscenico e schermo.

L'Eduardo giovane mostra verso il cinema un atteggiamento di curiosa «speculazione», nel senso - forse letterale - di riflessione operata a una certa distanza. Questa curiosità «distaccata» di Eduardo attraversa un periodo di formazione corrispondente ai secondi anni trenta e ai primi quaranta, ed è decisamente importante per come prolunga i suoi effetti nel cinema dei decenni successivi. Oltre che come attore, egli, già in tale periodo, è da considerare autore, se non ancora responsabile creativo del film in veste di regista, almeno nella veste di sceneggiatore-soggettista. Non è aspetto di poco conto, ma finora questa fisionomia e questo lato della carriera di Eduardo sono restati in sottordine, nell'ombra, e qui invece vanno ben sottolineati. L'attività di sceneggiatore comincia da Ma l'amor mio non muore, 1938, e giunge fino a Ti conosco mascherina!, 1943, passando per In campagna è caduta una stella, 1939, e Non mi muovo, 1943. Dei film diretti come regista, Eduardo in particolare dichiara di apprezzare non solo il primo, In campagna è caduta una stella ${ }^{4}$ a sua volta tratto da un copione

3. F. SAVIO, Cinecittà..., cit., p.409.

4. Su tale film, nell'intervista a F. SAVIO, Cinecittà..., cit., p. 408, Eduardo riconosce che egli passa dalla recitazione alla regia filmica perché «la regia mi interessava. E poi la storia di In campagna è caduta una stella non era male; non era teatro filmato, c'era aria, c'erano i paesaggi e la realtà della campagna». Poco dopo, nell'intervista che segue (SAVIO, Cinecittà..., cit., p. 411), si scopre, dalla testimonianza del fratello Peppino, che il soggetto di questo film è appunto di Peppino: «era un mio soggetto, tratto da una commedia mia, A Coperchia è cadu- 
di Peppino, ma anche il secondo, Ti conosco mascherina!, adattato da un testo di Scarpetta con mano e con grazia dinamica davvero encomiabile e sorprendente (almeno per me). ${ }^{5}$ In questo film si rivela un fine equilibrio degli elementi tipici della pochade e della farsa: travestimenti, equivoci, uso di maschere dialettali e di stereotipi comportamentali del teatro comico borghese. La tradizione del teatro s'avvera integralmente nelle dinamiche della commedia filmica e acquista il garbo di un divertissement sottile, penetrante, dove alla predizione iniziale del destino della protagonista (ella non potrà mai fare l'attrice, non ha talento, non ha la capacità di calcare la scena, perché «il teatro è una vocazione!» — dice un commendatore, regista di teatro, nella scena iniziale) corrisponde un finale con retrogusto amaro, un finto happy end (l'amore disinteressato della giovane donna viene sacrificato all'interesse pecuniario). Con tocco leggero e felice, Eduardo nel regime della commedia già esprime una vocazione drammatica, ma senza prevaricazioni, affidandosi all'intelligenza puntuale dei suoi spettatori.

Resta da marcare, per questo lato del contributo creativo da sceneggiatore, la duplicità nei confronti del fratello Peppino, che, spesso, accompagna il fratello Eduardo, pure lui, non solo in veste di attore e di co-protagonista dei film da ambedue interpretati (in questo periodo c'è soltanto l'eccezione di Una notte di fortuna, 1941, di Raffaello Matarazzo, regista col quale Peppino — che in questo film recita da solo, con Leda Gloria - si trova benissimo e che apprezza fino alla sua scomparsa). Peppino deve essere considerato anche per un preciso apporto creativo, da soggettista e sceneggiatore (talvolta non accreditato); capita a Eduardo di sviluppare soggetti del fratello Peppino e pure della sorella Titina (quest'ultima è autrice del soggetto di Ma l'amor mio non muore, 1938, diretto da Giuseppe Amato su sceneggiatura dei due fratelli e di Raffaello Matarazzo). A Peppino spetta una valutazione non subordinata a quella del fratello maggiore, restituendogli parità nella differenza, o meglio: uguale protagonismo nella duplice differenza che li tocca entrambi, inscindibilmente (vedi infra).

3. Nel dopoguerra Eduardo gioca, in modo più programmatico del decennio precedente, la carta della regia cinematografica. Egli attraversa la stagione del cinema di questo periodo — segnato da una vivacità industriale disomogenea e subalterna al potere degli statunitensi in Italia, da un forte ritorno dei generi popolari del comico e del dramma, del film in costume e avventuroso, e sul piano culturale dal rinnovamento estetico del neorealismo- con segni molto chiari: la sua opera di regista sembra iscriversi nell'alveo di una forma di dramma neorealista, specie con la versione di Napoli

ta una stella...». Ciò vale a dimostrare, ulteriormente, che in questo periodo nel cinema non è facilmente distinguibile il contributo creativo di Eduardo da quello di Peppino.

5. Su questo film, il giudizio di Eduardo è meno severo, riconosce che "non era male, una cosa graziosa»(F. SAVIO, ibidem), salvo poi constatare che il film comico in Italia avrebbe dovuto ricevere maggiori mezzi produttivi, oltre a richiedere più cura e più tempo del film drammatico, ma l'epoca lo impedì del tutto. 
milionaria, diretta nel 1950, primo film da lui firmato dopo la fine della seconda guerra. Eduardo innesta il suo dramma teatrale in una angolazione visiva chiaramente ispirata al neorealismo.

Ma si deve riconoscere che Napoli Milionaria (insieme a buona parte dell'esperienza filmica di Eduardo) va compresa dal lato di una delle significative anticipazioni del neorealismo, e non da effetto subordinato. Napoli Milionaria, cioè, pur iscrivendosi in una corrente che, da allora, marca irreversibilmente il cinema italiano, è esito stringente di uno dei presupposti del neorealismo. Eduardo, in altre parole, non segue una moda o una corrente perché ne è antesignano, essendo fra coloro che, da prima della guerra stessa, concorrono ad affermare la grande esperienza neorealista.

D'altro lato, la struttura del film mostra chiaramente che il cinema pensato e diretto da Eduardo pende irreversibilmente verso il melodramma o il dramma piccolo-borghese, dove la parola (quella presa in carico dai suoi personaggi e, in particolare, la «voce narrante») esprime come una distanza rispetto alle storie incorporate nei soggetti e nelle sceneggiature. La parola e la voce narrante sono una sorta di controcanto malinconico che talvolta spiazza lo spettatore, qualche volta lo accompagna in una resa didascalica delle immagini audiovisive e, non a caso, caratterizza l'immagine filmica nel segno di una evidente metafora «letterale». L'incipit e il finale di Napoli Milionaria sono espliciti e sintomatici di questo "difetto" di letteralità interpretativa che il testo sovraccarica sulle immagini e sulle loro capacità autonome di significare. È un pendant o ritornello concettuale che lo spettatore del dopoguerra ritrova in un altro suo importante film, diretto e interpretato: Napoletani a Milano, del 1953. Si tratta del film che Eduardo realizza dopo aver diretto una Filomena Maturano, 1951, dalla sua commedia, con Titina De Filippo; Ragazze da marito, 1952, e Marito e moglie, 1952.

L'esito di Napoletani a Milano non è scontato. E che sia una precisa scelta eduardiana è dimostrato, a mio avviso, da Marito e moglie, composto di due episodi, entrambi importanti ma per ragioni assai diverse; nel primo episodio - che personalmente preferisco — tratto da Eduardo, Diego Fabbri e Turi Vasile da una novella di Guy de Maupassant, si racconta la ben nota storia in cui il personaggio del marito, interpretato dallo stesso Eduardo, cova le uova, a ciò quasi costretto dall'implacabile, inflessibile e sterile moglie, Tina Pica. Questo episodio è come una promessa mancata di quel che Eduardo avrebbe potuto dare al nostro medium di immagini e suoni da una prospettiva interamente cinematografica; rimanda a una sorta di «felicità» dello stadio produttivo del film italiano del primo dopoguerra, dove alle segnature neorealiste (un bianco/nero documentario di spessore antropologico sulla comunità agricola fuori Napoli che si concentra sul miracolo delle uova) si accompagna la dimensione metaforica sul difficile e assai pungente registro delle relazioni uomo-donna, della sessualità e della procreazione, della sterilità femminile e del capovolgimento dei ruoli sessuali fra maschio e femmina. Ecco un Eduardo regista che dimostra di poter 
superare il limite fra teatro e cinema e dedicarsi a tempo pieno, con raggiunta maturità, alle strategie espressive del grande schermo. Ma il secondo episodio - pure importante nella sua caratura - sconfessa quel che il primo raggiunge; ricavato da un'opera teatrale del medesimo Eduardo, nella decorosa e pulita confezione torna al registro del dramma filmico essenziale e sigla l'impossibilità dell'autore di abbandonare il recinto e le radici creative del teatro moderno.

In Napoletani a Milano, Eduardo è regista e attore; vi interpreta il personaggio di un capogruppo che cerca soluzioni al disagio dell'emigrazione, e che tenta il reimpianto, nella maggiore città del Nord, dei fondamenti di comunità locale dialettale partenopea, usando più volte il refrain del tram che colleghi Napoli a Milano. Il refrain è l'espediente metaforico con cui l'autore intende esplicitare un sentimento didascalicamente «neorealista»; il tram che collega direttamente Napoli e Milano è l'immagine con cui la versione eduardiana della ricostruzione del paese, dopo la seconda guerra, vagheggia un volontaristico superamento, nella continuità quotidiana, delle differenze sociali e culturali sentite da coloro che soffrono i drammi dell'urbanizzazione e della crescita capitalistica nell'Italia del dopoguerra. Il film è generoso nell'intento, ma soffre di questa drammatizzazione popolare esibita e troppo pronunciata, senza farsi capacità autonoma, filmica, di mostrare e accendere scintille empatiche fra lo schermo e la sala. Questi limiti esibiscono un ritrarsi del genio di Eduardo rispetto al rinnovamento che il dopoguerra europeo impone, specialmente a coloro che hanno costruito la grande stagione del neorealismo, richiedendo la sperimentazione di innovativi schemi di racconto, nuove morali, scontri generazionali; nel cinema italiano, ciò comporta una radicale reiscrizione dell'esperienza neorealista in un sistema spettacolare complesso e differenziato, fra autori, generi popolari, contaminazioni fra intelligenze nazionali e immaginari sovranazionali.

4. Eduardo partecipa alla cultura italiana del dopoguerra e a quella cinematografica, sia in veste di regista di film tratti da sue opere - dopo Napoli Milionaria, una prima versione di Filumena Maturano, con Titina, nel 1951, Questi fantasmi del 1954 - o anche di commedie come Ragazze da marito, Marito e moglie e l'episodio L'Avarizia de I sette peccati capitali (tutti del 1952), Fortunella del 1958, Sogno di notte di una mezza sbornia del 1959, fino all'ultimo suo Spara più forte, più forte...non capisco del 1966; sia attraverso una serie di partecipazioni o collaborazioni con i maggiori rappresentanti del nostro cinema: Vittorio De Sica (L'oro di Napoli, Ieri oggi e domani), Roberto Rossellini (La macchina ammazzacattivi), Luigi Zampa (Campane a martello), Alessandro Blasetti (Tempi nostri. Zibaldone 2), Vittorio Cottafavi (Traviata '53), Gianni Franciolini (Villa Borghese e Ferdinando Io re di Napoli), Luciano Emmer (Le ragazze di Piazza di Spagna), Luigi Comencini (Tutti a casa), Antonio Pietrangeli (Fantasmi a Roma). A queste presenze davvero pregnanti, che confermano la solidarietà e l'impegno che Eduardo contrae con gli autori principali della commedia 
civile di costume nello scorcio del boom, si accompagnano altri film che, dal primo dopoguerra ai primi sessanta, testimoniano fasi puntuali della sua carriera filmica: in continuità con la commedia comica dell'anteguerra (dal Mario Mattòli di La vita ricomincia e Cinque poveri in automobile al Giuseppe Amato di Yvonne La Nuit), oppure nell'avanzare di un genere popolare drammatico alla Matarazzo, con ambizioni sociologiche e documentariste (dal Glauco Pellegrini di L'amore più bello all'Antonio Petrucci di Cortile), o ancora nella commedia di costume che transita nella satira e nel film civile (dal Giorgio Pàstina di Cameriera bella presenza... a Lionello De Felice di Cento anni d'amore, ai sopracitati film di Emmer, Comencini, Pietrangeli).

Questo panorama vasto e articolato richiede alcune distinzioni che rendano giustizia al ruolo — strategico — che Eduardo svolge accompagnando alcuni snodi della carriera dei maestri del cinema del dopoguerra. Mi riferisco soprattutto a Rossellini e a De Sica, in rapporto ai quali si deve separare l'Eduardo-attore dall'Eduardo-autore. Ed è su questo secondo che vale la pena di riflettere, anche se ciò voglia dire smettere per un po' di trattare direttamente Eduardo per indagare, piuttosto, l'opera di quei due maestri del cinema italiano.

L'incontro dell'Eduardo-autore con Rossellini è l'esito di un rapporto di stima e di ammirazione reciproca, ma anche complesso, che ratifica la loro profonda diversità nella visione del cinema. Anche l'incontro con De Sica, pur avendo alla base un tratto comune di origine - gli inizi di carriera con Mario Camerini e con il modello di una commedia drammatica lucida e pungente - risulta problematico, giungendo a soluzioni controverse e non sempre condivise dai due.

Luso del termine strategico per definire il rapporto di Eduardo con questi due maestri va inteso anzitutto nel senso di innesco creativo, di promozione e scelta verso elaborazioni filmiche che senza Eduardo probabilmente non sarebbero state concepite. Contributo strategico, quindi, dal punto di vista dell'ideazione: elemento positivo senz'altro ricondotto al genio di Eduardo, alla capacità di leggere la realtà italiana e napoletana e di rimetterla in opera e trama drammatica e narrativa. Ma, non tanto paradossalmente, risulta di converso strategica anche la distanza che le opere filmiche di Rossellini e De Sica conquistano rispetto all'idea originaria di Eduardo: un elemento dunque negativo che, mentre evidenzia come Eduardo resti al di qua del territorio cinematografico nel quale i due registi riversano creatività ed estro espressivo, consente di misurare le conquiste che questi due maestri apportano al cinema italiano del dopoguerra.

Rossellini realizza nel 1948 La macchina ammazzacattivi da un soggetto di Eduardo e di Filippo Sarazani. Il film è stato variamente interpretato e qui non ripercorro le caratteristiche salienti che lo rendono chiaramente un film di transizione. Mi limito a dedurre l'interesse specifico di Rossellini per questo soggetto eduardiano. Ce ne è uno "esterno": l'amore di Rossellini per la costiera amalfitana, luogo nel quale girare tale soggetto gli dà 
modo di vivere una sorta d'armonia edenica, fatta di aria, luce e odori naturali fra mare e costa, che il grande regista persegue come sua cifra personale di vita (sulla quale vi sono molte testimonianze; per questo stesso motivo, La macchina ammazzacattivi non è l'unico film rosselliniano girato in costiera). Ma c'è un interesse di Rossellini «interno» alla dimensione tematica che il soggetto narrativo di Eduardo gli consente di trattare: la costiera amalfitana è un paradiso terrestre che diviene d'improvviso luogo di una dannazione infernale resa possibile dalla macchina fotografica! Il soggetto di Eduardo, in altri termini, evidenzia il ruolo decisivo di una mutazione fantastica: uno spunto narrativo che, dalla fiaba, arriva all'apologo sociale, e che penetra nella qualità nascosta e sedimentata dell'attrezzatura tecnica del cinema, colto nella capacità di congiungere e collegare corpo e anima.

Il film manifesta una particolare riflessione sulla duplicità intrinseca alla fotografia dinamica della realtà che, ideologicamente, le teorie del neorealismo spesso commettono l'errore di ridurre alla sola ostentazione visiva e fenomenologica di ciò che si predispone frontalmente alla macchina da presa. Da tale prospettiva, il soggetto eduardiano, per come esordisce già dal prologo (una commedia, viene detto dalla voce di un demiurgo che, dal fuori campo, dalle nebulose dell'inquadratura, inscena un teatrino immobile che sfoca nel nero da cui parte l'immagine della costiera e di un auto con americani a bordo) promette molto per Rossellini. Esso gli dà modo, in forma chiaramente metaforica, di uscire fuori dalle strettoie ideologiche in cui, nel 1948, si classifica il movimento neorealista; La macchina ammazzacattivi rivendica una complessità in grado di toccare le molteplici dimensioni di significato e di comunicazione gestibili dall'immagine filmica: dal sacro al profano, dal teatro al cinema, dalla fiaba alla denuncia civile, dalla profezia alla commedia, dal documentario al narrativo. Rossellini coglie nella dimensione autoriale di Eduardo una vocazione transmediale (non soltanto metaforica) che gli apre orizzonti meno univoci e dà fiato alla sua poliedrica, polivalente, capacità di innovare il cinema in ogni direzione, in un momento decisivo dell'evoluzione del panorama filmico italiano nel secondo dopoguerra.

A sua volta, Vittorio De Sica incontra artisticamente Eduardo (che da attore conosce dagli anni trenta) in una fase avanzata della sua carriera di regista. Si tratta dei primi anni sessanta, quando De Sica traspone sullo schermo soggetti o testi teatrali di Eduardo, prima con l'episodio Adelina di Ieri, oggi e domani nel 1963, poi con Matrimonio all'italiana, dell'anno successivo, che è la sua celebre versione di Filumena Maturano. Questi due film —entrambi interpretati da Sofia Loren - sono momenti importanti del cinema di De Sica nel corso degli anni sessanta, un periodo nel quale ha alle sue spalle non solo i suoi massimi capolavori del primo dopoguerra (Ladri di Biciclette, 1948, Miracolo a Milano, 1951, Umberto D, 1952, L'oro di Napoli, 1954), ma anche film come il Boom, 1963 e I sequestrati d'Altona, 1962. 
Con questi film, De Sica s'impone - perfino dopo l'insuccesso de Il giudizio universale, 1961, bilanciato dal grande successo di La ciociara e dall'Oscar a Sofia Loren nel 1960 - come uno dei registi di maggiore incasso nel nostro paese; i produttori gli affidano progetti impegnativi in termini di risorse. De Sica sa sempre economizzare, girare in modo veloce e preciso nei risultati e negli obbiettivi, conseguendo risultati davvero straordinari come I girasoli, 1969, Il giardino dei Finzi Contini, 1970, Una breve vacanza, 1973, fino all'ultimo suo bel film, Il Viaggio, del 1974.

De Sica è interessante per l'indagine sull'Eduardo soggettista-sceneggiatore perché esistono pagine da lui scritte, mentre gira i due film sopra citati, che non solo riconducono allo straordinario rapporto che il regista vive con Napoli e i napoletani, ma anche al lavoro propriamente cinematografico svolto sui soggetti eduardiani. Adelina proietta lo spettatore in una Napoli poverissima, popolare, «antica»: Adelina (che ha le prorompenti grazie femminili di Sofia Loren) è una donna dai molti figli, vende sigarette di contrabbando, vive in un "basso" assai povero e spoglio e, con la complicità del marito (Mastroianni), per sfuggire alla carcerazione (non ha pagato una multa con diverse, più gravi, conseguenze) si fa ingravidare regolarmente, godendo del beneficio d'aspettativa riservato alle donne incinte; finché il marito ha una defaillance fisica e, quindi, lei non può sottrarsi al carcere. La testimonianza di De Sica è straordinariamente vivace in merito alle mille vicissitudini trascorse nel girare tra i vicoli di Napoli e del rione Sanità. ${ }^{6} \mathrm{Ma}$ ha modo, anche, di specificare alcune cose circa il soggetto di Eduardo: da un lato, riportandosi alla scena in cui Sofia viene a sapere che esce dal carcere, apprezza una battuta che Adelina pronuncia ("I m'e mangio, m'e mangio»), battuta "che è una vera intuizione poetica di Eduardo»; dall'altro, descrivendo come sta dirigendo la scena della camerata delle donne madri, De Sica non può fare a meno d'osservare che «il pericolo di questa sceneggiatura di De Filippo sta nello stile un po' antiquato, per nulla cinematografico. Ogni scena è una specie di bozzetto teatrale. Io cerco con la macchina di dargli una meccanica moderna fatta di movimenti, di angolazioni diverse, ma le azioni, i dialoghi hanno, per loro natura, impostazione teatrale. Aggiungerò, ai momenti interni che ci sono, esterni, in modo che Napoli sia sempre presente e i napoletani facciano da coro». ${ }^{8}$

De Sica pare quindi assumere la sceneggiatura di Eduardo come un canovaccio o idea narrativa globale, di valore poetico, da cui espungere il trattamento e la concezione formale di impianto teatrale, che gli risulta statica; sul piano recitativo, anzi, caricata eccessivamente e, pertanto, inadatta al movimento del cinema e alle sue angolazioni foto-incisive.

6. V. DE SICA, Lettere dal set, Milano: SugarCo Edizioni, 1987.

7. V. DE SICA, cit., p. 114.

8. V. DE SICA, cit., p. 115. Più avanti, p. 148, De Sica, mentre attende di vedere il materiale girato a Napoli, annota: «sono molto curioso di vedere di quale gusto e di quale stile sono tutte le scene che io ho aggiunto alla teatralissima sceneggiatura di De Filippo; spero vadano bene» (corsivo mio). 
In alcuni passi dei diari quotidiani sulla lavorazione di Matrimonio all' taliana, De Sica esprime un analogo e più deciso giudizio. In un passo delle sue lettere alla figlia Emi, prende una chiara posizione: «Leggo sul giornale che De Filippo scrive che la sceneggiatura non è sua e che la responsabilità ricade tutta sugli altri collaboratori. La sua sceneggiatura non è stata accettata da me. Vorrei fare lettura pubblica di quella sceneggiatura. Fortunatamente abbiamo preso le redini io e Tonino Guerra ed abbiamo potuto girare questo film, altrimenti avremmo dovuto rinunciares. ${ }^{9}$ In questo passaggio, va notato che sia De Filippo che De Sica parlano di sceneggiatura e non di testo teatrale (Filumena Marturano). Eduardo deve anche lui aver lavorato allo script destinato a ricavare un film dalla sua opera teatrale, ma tenendo in piedi una formulazione visiva che De Sica deve aver ritenuto insufficiente, inefficace. Tant'è che, in relazione a una delle scene più significative — «Filumena di fronte ai figli e la confessione di essere la loro mamma» - De Sica, nuovamente, scrive di una precisa differenza del suo modo di concepire l'immagine filmica rispetto a Eduardo: «il pericolo della scena era che era tratta di peso dalla commedia di De Filippo e Sofia doveva misurarsi con l'interpretazione che ne fece la povera Titina De Filippo. In più, il tono, l'impostazione della scena sono di carattere assolutamente teatrale e togliere la teatralità è stato per me un compito arduo». ${ }^{10}$

De Sica insomma rispetta la poetica del grande autore napoletano, ma la inscrive in coordinate che riscattino la capacità del cinema di penetrare a fondo nel dramma di una madre e del suo rapporto con i figli e con l'uomo da sempre amato, senza le sottolineature del gesto carismatico della scena, o del dialogo sufficiente a se stesso, ma nella puntuale insorgenza del moto dell'anima riflesso nel gesto o nella fisionomia minimale del volto, catturato dalla macchina da presa attraverso un lavoro paziente, severo, puntuale, quasi ossessivo nella ripetizione dei ciak che cercano di acciuffare emozioni altrimenti imprendibili.

E basta questo spunto di analisi, per mostrare come il film di De Sica risolve sullo schermo un insieme di limiti e di diaframmi che, puntualmente, si frappongono fra l'universo creativo eduardiano, di natura e concezione prettamente teatrali, e le modalità espressive del cinema. Una indagine completa del film di De Sica — dispiegando diffusamente segni e fatti espressivi che «risolvono» e inquadrano questioni relative allo spinoso rapporto fra scena teatrale e schermo filmico- avrebbe qui il demerito di condurre davvero lontano dal tema da trattare, che resta Eduardo e la sua opera.

5. Un apporto davvero importante alla comprensione del titolo e del merito di questo contributo, «il cinema idea di Eduardo» si ricava dalle riflessioni svolte da parte di Orson Welles sul tema dell'attore cinematografico e sul rapporto che questo contrae con la macchina da presa. Il pensiero espres- 
so da Welles sull'attore nel cinema ben presto incrocia la figura di Eduardo e la prospetta da esempio sommamente "critico». L'incontro fra Welles e Eduardo sorprende soltanto chi dimentica che entrambi furono uomini di teatro e di sperimentazione nel corso del Novecento; senz'altro Welles vede e apprezza Eduardo nel corso degli anni trascorsi a Roma, fra la fine dei cinquanta e i primi settanta, e non deve quindi stupire se in alcune testimonianze e interviste Welles faccia non casualmente alcune valutazioni su Eduardo.

Queste sono contenute, in particolare, in un breve passo delle interviste di Peter Bogdanovich ${ }^{11}$ al grande regista americano. Welles fa un esplicito riferimento a Eduardo ${ }^{12}$ per argomentare sulla natura e sulle caratteristiche dell'attore nel cinema. Il grande regista considera Eduardo come attore di teatro e poi di cinema dopo aver riflettuto in generale sull'attore in scena o sullo schermo. Dapprima, il suo pensiero prende a esempio Gary Cooper ("Lo vedevi lavorare sul set e pensavi: "Dio mio, questa dovranno rigirarla!". Praticamente sembrava che non ci fosse. E poi vedevi i giornalieri, e riempiva lo schermo»), ${ }^{13}$ ritenuto caso classico dell'attore cinematografico che s'impone — dice Welles - per la «Personalità. Non presumo di risolvere questo mistero. Ma conta sempre più della tecnica». ${ }^{14}$ Da qui Welles subito considera Laurence Olivier: «...chi conosce la tecnica meglio di Olivier? Certo che se la recitazione cinematografica dipendesse in modo significativo da una tecnica particolare, Larry se ne sarebbe impadronito. Eppure, per quanto bravo sia al cinema, è solo un'ombra dell'attore che impone la sua presenza magnetica sulle scene teatrali. Perché la macchina da presa sembra diminuirlo? E ingrandire Gary Cooper, che di tecnica non ne sapeva un bel niente?» ${ }^{15}$

Welles imposta il suo ragionamento sull'attore nel cinema inquadrandone il mistero rispetto a figure — pur grandissime - che in teatro hanno conquistato la scena classica e moderna. Dopo aver passato in rassegna la figura di Scialapin («...il cui genio era pari all'enorme statura della sua personalità»; "... nessuno è della sua categoria. E che cos'è sullo schermo? Sì, resta un po' impresso, e basta») ${ }^{16}$ e quella di Frank Fay («il più grande degli attori di vaudeville...sullo schermo era un fantasma») ${ }^{17}$ Welles mette a fuoco quel che egli nomina «un perfetto esempio di questo mistero: Eduar-

11. P. Bogdanovich, Io, Orson Welles, Firenze: Baldini\&Castoldi, 1993.

12. P. BogdANOVICH, Io ..., cit., p. 21, svela che l'aneddoto relativo a Eduardo (che cioè lo stesso Bogdanovich "sia uscito dallo spettacolo di De Filippo a Roma») in realtà è una pura invenzione di Welles, il quale — riscrivendo la bozza dell'intervista di Bogdanovich — ha inserito un fatto mai accaduto; quel che conta, per noi, è che Welles abbia voluto considerare Eduardo un esempio decisivo — al di là del riferimento a un fatto realmente successo- entro un ragionamento teso a chiarire cosa sia l'attore nel cinema.

13. P. BogdanOvich, Io.., cit., p. 49.

14. Ibidem.

15. P. Bogdanovich, Io..., cit., p. 48-49.

16. P. BogdanOVICH, Io..., cit., p. 49.

17. Ibidem. 
do De Filippo. Sulla scena non c'è nessuno che gli si possa anche solo avvicinare. Al cinema non c’è più»». ${ }^{18}$

La riflessione su Eduardo attore sullo schermo ricalca in questi passaggi wellesiani certe notazioni già avanzate con un occhio sommario (talora colpevole) da vari osservatori. Assumere qui il punto di vista wellesiano vale non solo per la sua autorità, e nemmeno in quanto la sua valutazione non è affatto diminutiva della figura e della enorme bravura di Eduardo; ${ }^{19}$ ma perché Welles propone una spiegazione convincente, esaustiva, intrigante, della differenza che qui intendo cogliere.

Di seguito, infatti, la riflessione di Welles si inerpica su cosa fa la macchina da presa sull'attore. E, dopo aver riportato una spiegazione "semplice» di Akim Tamiroff, per il quale la macchina da presa ama o non ama gli attori (in rapporto a Eduardo, ne deriva la conclusione che «La macchina da presa non lo ama»); ${ }^{20}$ dopo aver sgombrato il campo da osservazioni non pertinenti sul recitare sopra o sotto le righe («la macchina da presa non ti obbliga a recitare sotto le righe [...] sei tu che metti i limiti, non la macchina da presa»), ${ }^{21}$ Welles va al cuore di un concetto decisivo: "Quello che la macchina registra è l'assenza o la presenza del sentire [...] La forza emotiva può investire il pubblico di un teatro, ma sullo schermo spesso è difficile tenerla a fuoco. [...] Quel che fa la macchina da presa, ed è l'unica cosa che fa, $\grave{e}$ fotografare il pensiero [...] La macchina da presa non è tanto una macchina della verità, quanto un contatore Geiger di energia mentale. Registra qualcosa che l'occhio nudo distingue solo vagamente, scorgendone qualche traccia, e te lo registra forte e chiaro: registra il pensiero. Ogni volta che un attore pensa, nel film si vede [...] La macchina da presa è un critico». ${ }^{22}$

Queste straordinarie riflessioni di Welles circoscrivono chiaramente il cinema-idea di Eduardo: una presenza attoriale sullo schermo che fa registrare un distacco fra l'attore e i personaggi interpretati, una distanza che la m.d.p. - dice Welles- «registra forte e chiaro». O, anche, una sapienza interpretativa che, tuttavia, viene restituita come traccia mnemonica o recita-

18. Ibidem.

19. La stima di Orson Welles nei confronti di Eduardo è forse anche alla base della partecipazione del grande attore napoletano a un film oggi quasi dimenticato, Raw Wind in Eden, Vento di passioni, con Esther Williams, Jeff Chandler e Rossana Podestà, diretto nel 1958 proprio da quel Richard Wilson che è stato, fino alla recente scomparsa, l'assistente, il collaboratore più assiduo —oltre che biografo e memoria vivente e conservatore integrale- dell'opera di Orson Welles. Noto qui — di passaggio — che dovrebbero meglio ricostruirsi i fili intrecciati e complessi che, fra teatro e cinema, legano le due grandi figure di Welles e Eduardo a Laurence Olivier: fili che, a loro volta, legano Eduardo a due fra i massimi interpreti novecenteschi del teatro e del cinema shakespeariano.

20. Ibidem. È in ogni caso divertente immaginare come lo stesso Welles rifaccia, da attore, davanti al suo interlocutore, Peter Bogdanovich, la voce e il modo di parlare di Akim Tamiroff: "Machina guarda tipo uno", dice, e machina dice: 'Zì̀̀, guesto me piace!, Machina guarda tipo due, machina dice, 'Noooo! Ghi sape perche machina? Nesuno sape» (ibidem).

21. P. Bogdanovich, Io..., cit., p. 50.

22. P. Bogdanovich, Io.., cit., p. 50-51. 
zione irrigidita nella tecnica. Ecco determinata la posizione singolare, complessa e difficile, che un attore di tale levatura conquista nella galleria delle figure che si muovono entro le coordinate dell'attore cinematografico. Il giudizio wellesiano non è senza esiti per il compito di ricostruire i fondamenti caratterizzanti della figura attoriale di Eduardo, dagli inizi della sua carriera nel cinema fino alle sue ultime performance negli anni sessanta. Consente un avvicinamento sapiente al cuore di quella duplice differenza (o duplicità differente) che l'Eduardo attore assume, in modo estremamente preciso, nei confronti della figura del fratello, il Peppino attore.

6. Dall'inizio alla fine, Eduardo e Peppino mostrano nel cinema di essere duplici l'uno all'altro, con segnature che ne differenziano la posizione. Una comparazione fra i due De Filippo dovrebbe ripercorrere specialmente, ma non solo, i film recitati insieme in gioventù, negli anni trenta (per esempio, Quei due, 1935, di Gennaro Righelli; Sono stato io!, 1937, di Raffaello Matarazzo; L'amor mio non muore, 1938, di Giuseppe Amato e Mario Bonnard). Il loro primo film è Tre uomini in frak, di Mario Bonnard nel 1932. Con Il cappello a tre punte, diretto da Mario Camerini nel 1935, i due De Filippo s'impongono nella memoria degli spettatori italiani di quel periodo. ${ }^{23} \mathrm{Il}$ film non solo segna un salto di qualità nella carriera filmica da poco iniziata dei due fratelli, ma ha il particolare merito di evidenziare i segni costitutivi della filigrana recitativa con cui Peppino e Eduardo vicendevolmente edificano la propria presenza attoriale nel cinema, imponendo una reciproca, differente singolarità.

Senza entrare nel merito analitico del film di Camerini e sul ruolo davvero centrale che vi occupa la duplice caratterizzazione dei due fratelli, ${ }^{24}$ occorre sinteticamente ricapitolare come il cinema-idea di Eduardo sia già in esso attivamente presente, paragonando la duplicità che vi emerge fra Eduardo e Peppino con quella riproposta da uno degli ultimi film recitati dai due fratelli insieme, in ruoli antagonisti, quasi trent'anni dopo: Ferdinando Io Re di Napoli, 1959, di Gianni Franciolini.

Nel film di Camerini, l'antagonismo fra Peppino e Eduardo vede il primo incarnare un eroe d'azione che muta in sapiente personaggio da commedia (un fornaio che si traveste da Governatore per vendicare l'oltraggio subito dalla giovane moglie), mentre il secondo rappresenta una maschera (un Governatore che non riesce a essere efficacemente seduttivo e rientra infine nel ruolo dell'esponente del potere disincarnato dell'autorità rivestita). Tuttavia l'antagonismo, salendo di grado fino a essere vera e propria duplicità, non è rigido; passa, anzi, attraverso ogni contaminazione e ogni registro simbolico-narrativo. La duplicità raddoppia le recipro-

23. Il film di Camerini decretò il successo cinematografico dei due fratelli presso il grande pubblico delle sale cinematografiche. Cfr. ciò che entrambi dichiarano nelle interviste di F. SAVIO, Cinema anni trenta, vol. I, Roma: Bulzoni, 1974, p. 407-415.

24. Per una analisi specifica, rimando al mio saggio «Peppino eroe del cinema», in P. SABBATINO e G. Scognamiglio (a cura di), Peppino e la comicità nel Novecento, Napoli: Edizioni Scientifiche Italiane, 2005, p. 169-180. 
che funzioni assunte dai due fratelli, anche simbolicamente: ciascuno dei due prende i panni e «usurpa» le apparenze e i ruoli dell'altro. Ma la duplicità è, pure, alterità, differenza incolmabile, contrasto che alimenta il confronto e sostanzia la contrapposizione. È una partita a due, nutrita da ogni variazione consentita dai poli estremi che ciascuno dei fratelli occupa, riservandosene l'esclusiva.

Nel film di Franciolini, realizzato qualche decennio dopo, la duplicità Eduardo/Peppino si dichiara nuovamente: fra il Re Lazzarone/Peppino e il suo più acerrimo avversario, un Pulcinella/Eduardo che scrive una canzone che rima in burla il Re stesso e le sue avventure amorose. La comparazione fra uno dei primissimi e uno degli ultimi film interpretati insieme dai due De Filippo, a distanza di ventiquattro anni, dal 1935 al 1959, ricalca una autentica posta in gioco, che trascorre e si evolve per il lungo periodo della competizione attoriale dei fratelli.

Mentre ne $I l$ cappello a tre punte si dispone e si afferma, per la prima volta, la differenza fra la maschera/volto di Eduardo - la sua recitazione quasi tutta mentale - e la flagranza dal ritmo carnale di Peppino (che investe l'intero corpo: dal volto alle mani, dal gesto minimo alla figura intera, alla voce che esprime l'irruenza del sentimento), in Ferdinando Io Re di Napo$l i$, questa differenza si ripropone tematicamente nel soggetto narrativo: Peppino è il Re Lazzarone che seduce e va a caccia di belle donne, rivendicando nella sua doppia identità di «guappo» le ragioni di una auspicata e sempre tentata felicità del corpo; mentre Pulcinella/Eduardo, che riveste proprio la parte di un attore e capocomico teatrale, scrive canzoni e persegue utopie rivoluzionarie, assegnando a sé lo spazio, la metafora, dell'idea di libertà.

Ecco gli estremi su cui i due fratelli si posizionano: Peppino è un corpo/immagine che si dà nella completa performance attoriale e in essa esaurisce ogni significazione; mentre Eduardo quasi trattiene il corpo per esprimere una idea/immagine che sta fuori da sé, come a rintracciare una radice critica, razionale, tradizionale, in cui riconoscersi. Mentre Peppino si fa cinema (nell'unione inscindibile corpo-parola-voce), Eduardo cerca nel cinema qualcosa che (nella scissione fra corpo-parola-voce, o nella loro interdizione o blocco) lo riconduca in una dimensione - quella del Teatro- alla quale sente, maggiormente, di appartenere.

\section{Indicazioni bibliografiche essenziali}

G. FreZZA, Napoli nel Novecento fra cinema e televisione, in A. Croce, F. Tessitore, D. CONTE (a cura di), La Cultura a Napoli e in Campania nel Novecento, vol. II (a cura di M. PAlumbo), Napoli: Liguori, 2007.

P. IACCIO (a cura di), «Napoli e il Cinema (1896-2000)», Nord e Sud, nuova serie, A. 47, n. 4, 2000, Napoli: Edizioni Scientifiche Italiane.

P. Bogdanovich, Io, Orson Welles, Firenze: Baldini\&Castoldi, 1993.

M. ARGENTIERI (a cura di), Risate di regime. La commedia italiana 1930-1944, Venezia: Marsilio, 1991. 
VitTorio De SicA, Lettere dal set, Milano: SugarCo Edizioni, 1987.

G.P. BRUNETTA, Storia del cinema italiano, vol. I: $1895-1945$ e vol. II: Dal 1945 agli anni ottanta, Roma: Editori Riuniti, 1979-1982.

S. GrmeK Germani, «Mario Camerini», Il castoro cinema, n. 84, Firenze: La Nuova Italia, 1980.

F. SAVIO, Cinecittà anni trenta, Roma: Bulzoni, 1979, 3 vol.

A. APRA - P. Pistagnesi (a cura di), Nuovi materiali sul cinema italiano 1929/43, Pesaro: Mostra Internazionale del Nuovo Cinema, 1976, 2 vol.

F. SAVIO, Ma l'amore no, Milano: Sonzogno, 1975.

A. APRA (a cura di), Materiali sul cinema italiano 1929/43, Pesaro: Mostra Internazionale del Nuovo Cinema, 1975. 